\title{
Shape sensing of aerospace structures by coupling of isogeometric analysis and inverse finite element method
}

\author{
Adnan $\mathrm{Kefal}^{1}$ and Erkan Oterkus ${ }^{2}$ \\ University of Strathclyde, 100 Montrose Street, Glasgow G4 OLZ, UK
}

\begin{abstract}
This paper presents a novel isogeometric inverse Finite Element Method (iFEM) formulation, which couples the NURBS-based isogeometric analysis (IGA) together with the iFEM methodology for shape sensing of complex/curved thin shell structures. The primary goal is to be geometrically exact regardless of the discretization size and to obtain a smoother shape sensing even with less number of strain sensors. For this purpose, an isogeometric KirchhoffLove inverse-shell element (iKLS) is developed on the basis of a weighted-least-squares functional that uses membrane and bending strain measures consistent with the KirchhoffLove shell theory. The novel iKLS element employs NURBS not only as a geometry discretization technology, but also as a discretization tool for displacement domain. Therefore, this development serves the following beneficial aspects of the IGA for the shape sensing analysis based on iFEM methodology: (1) exact representation of computational geometry, (2) simplified mesh refinement, (3) smooth (high-order continuity) basis functions, and finally (4) integration of design and analysis in only one computational domain. The superior capabilities of iKLS element for shape sensing of curved shells are demonstrated by various case studies including a pinched hemisphere and a partly clamped hyperbolic paraboloid. Finally, the effect of sensor locations, number of sensors, and the discretization of the geometry on solution accuracy is examined.
\end{abstract}

\section{Introduction}

Over the last few decades, Structural Health Monitoring (SHM) has become a key technology of the future aerospace structures to achieve significant improvement in safety, reliability, and affordability. A typical SHM system provides real-time vehicle health information via sensors and software to mitigate accidents due to failure and achieve safe landing. The inverse Finite Element Method (iFEM) is a state-of-the-art methodology originally introduced by Tessler and Spangler [1,2] for real-time reconstruction of full-field structural displacements in plate and shell structures that are instrumented by strain sensors. This inverse problem, commonly known as shape sensing, is the most crucial component of a typical SHM system. Since the first publication of iFEM algorithm [1,2], many different numerical and experimental studies have been devoted to expand the horizon of the iFEM methodology in the literature. For instance, Tessler and Spangler [3] developed a three-node inverse shell element (iMIN3) utilizing lowest-order anisoparametric $\mathrm{C}^{0}$-continuous shape functions and adopting the kinematic assumptions of the Mindlin's plate theory. More recently, Kefal and co-workers [4] have recently formulated a four-node quadrilateral inverse-shell element, iQS4, based upon a weighted-least-squares functional. This new element includes hierarchical drilling rotation degrees-of-freedom (DOF) and further extends the practical usefulness of iFEM for shape-sensing analysis of large-scale structures. Moreover, Gherlone and co-workers [5] formulated a robust inverse frame element that uses kinematic assumptions of Timoshenko beam theory including stretching, bending, transverse-shear and torsion deformation modes. Furthermore, Cerracchio et al. [6] has recently improved the original iFEM formulation Tessler and Spangler [1,2] by adding the kinematic assumptions of recently developed Refined Zigzag Theory [7] in order to perform SHM of multi-layered composite and sandwich structures. Apart from theoretical developments, Kefal and Oterkus [8] performed shape-sensing of a longitudinally and transversely stiffened plate as a fundamental application of the iFEM framework to SHM of marine structures. Similarly, Kefal and Oterkus [9] presented a more sophisticated application of iFEM to marine structures namely displacement and stress monitoring of a chemical tanker based on iFEM algorithm. More recently, Kefal and Oterkus [10] have demonstrated the application of the iFEM methodology

\footnotetext{
${ }^{1} \mathrm{PhD}$ Researcher, Department of Naval Architecture, Ocean, and Marine Engineering.

${ }^{2}$ Associate Professor, Department of Naval Architecture, Ocean, and Marine Engineering, AIAA Member.
} 
for monitoring multi-axial deformations and stresses of a Panamax containership advancing in beam sea waves. All these investigations have so far proved that the iFEM framework is an accurate, robust, and fast shape- and stresssensing algorithm

Moreover, isogeometric analysis (IGA) originally introduced by Hughes et al. [11], presents smooth function spaces such as Non-Uniform Rational B-spline (NURBS) to numerically solve a number of engineering problems. Recently, IGA received a great deal of attention from both academy and industry (e.g., refer to [12]) due to the highorder continuity $\left(C^{p}, p>0\right)$ provided by NURBS in both geometric domain and physical simulation. In fact, a smoother shape sensing can be obtained based on the iFEM framework, if the shape functions ensure a high-order continuity throughout the element interior and edge interface. Therefore, the main and novel aim of this study is to carry out a better shape sensing analysis of aerospace structures by coupling the NURBS-based IGA together with the iFEM methodology. For this purpose, a rotation-free isogeometric inverse-shell element is developed based on the kinematic assumptions of the Kirchhoff-Love plate/shell theory and this inverse-shell element labelled as iKLS. The shape sensing capability of the iKLS element is validated by solving two numerical examples, namely pinched hemisphere and partly clamped hyperbolic paraboloid. Finally, the effect of sensor locations, number of sensors and the discretization of the geometry on solution accuracy is examined.

\section{Rotation-free isogeometric inverse-shell element formulation}

A novel isogeometric Kirchhoff-Love inverse-shell element, labeled iKLS, is developed on the basis of iFEM least-squares formulation. This development couples the NURBS-based IGA together with the iFEM methodology for shape sensing analysis of thin shell structures such as aerospace structures. In the following formulation, Greek indices take the values of 1 and 2 while the Latin indices range from 1 to 3 . The convective coordinates are $\boldsymbol{\beta}=\left[\begin{array}{lll}\beta^{1} & \beta^{2} & \beta^{3}\end{array}\right]^{T}$ where $\beta^{\alpha}$ denotes in-plane coordinates and $\beta^{3}$ defines thickness direction of the shell. The iKLS element has a uniform shell thickness $\beta^{3} \in[-h,+h]$ and the material points located at the mid-surface of the shell are described as $\beta^{3}=0$. The parametric coordinates $\xi=\left[\begin{array}{lll}\xi & \eta & \zeta\end{array}\right]^{T}$ used to define the NURBS basis functions are associated with the convective coordinates $\boldsymbol{\beta}$ as physical space. The necessary position vectors to develop the iKLS formulation, translation degree-of-freedoms (DOFs) of $i^{t h}$ control point $\left(d_{1}^{i}, d_{2}^{i}, d_{3}^{i}\right)$, and displacement vector components $\left(v_{1}, v_{2}, v_{3}\right)$ are demonstrated in Figure 1.

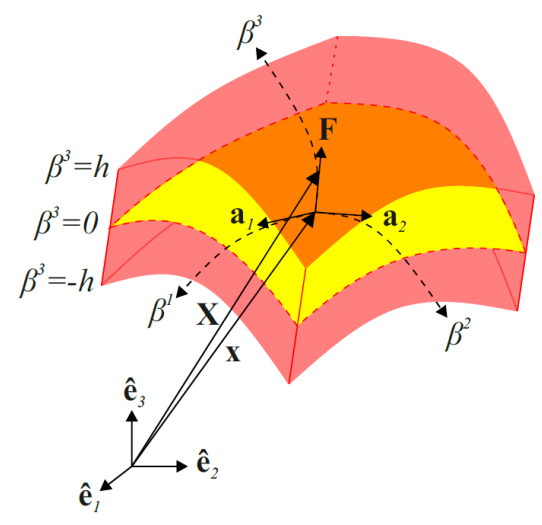

(a)

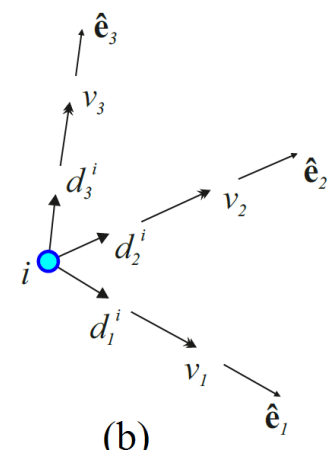

(b)

Figure 1. (a) iKLS element. (b) Translational DOFs of $i^{\text {th }}$ control point.

The position vector to a material point on the mid-surface of the iKLS element can be described by the finite sum of NURBS element basis functions as

$$
\mathbf{x} \equiv \mathbf{x}\left(\beta^{1}, \beta^{2}\right)=\sum_{i} R_{i}(\xi, \eta) \mathbf{B}_{i} \equiv \sum_{i} R_{i} \mathbf{B}_{i}
$$


where $R_{i}$ is the NURBS basis functions and $\mathbf{B}_{i}$ are the coordinates of the control points that defines the physical geometry of the iKLS element. The covariant base vectors of mid-surface $\mathbf{a}_{\alpha}$ can be obtained in reference configuration by taking the partial derivatives of $\mathbf{x}$ with respect to convective coordinates $\beta^{\alpha}$ as

$\mathbf{a}_{\alpha} \equiv \mathbf{a}_{\alpha}\left(\beta^{1}, \beta^{2}\right)=\frac{\partial \mathbf{x}}{\partial \beta^{\alpha}}=\sum_{i} R_{i, \alpha} \mathbf{B}_{i}$

Using the basis vectors $\mathbf{a}_{\alpha}$, a unit-magnitude vector field (the director vector) that is perpendicular to the tangent plane of any point belongs to mid-surface can be defined as

$\mathbf{F} \equiv \mathbf{F}\left(\beta^{1}, \beta^{2}\right)=\frac{\mathbf{a}_{1} \times \mathbf{a}_{2}}{\left\|\mathbf{a}_{1} \times \mathbf{a}_{2}\right\|}$

Then, the position vector $\mathbf{x}$, the director vector $\mathbf{F}$, and the thickness direction convective coordinate $\beta^{3}$ can be utilized to define a position vector to an arbitrary material point of the shell body in the reference configuration as

$$
\mathbf{X} \equiv \mathbf{X}\left(\beta^{1}, \beta^{2}, \beta^{3}\right)=\mathbf{x}\left(\beta^{1}, \beta^{2}\right)+\beta^{3} \mathbf{F}\left(\beta^{1}, \beta^{2}\right)
$$

The displacement vector $\mathbf{v}$ representing the displacement field between reference and current configurations of the shell body can be defined as

$$
\mathbf{v} \equiv \mathbf{v}\left(\beta^{1}, \beta^{2}\right)=\left[v_{1}\left(\beta^{1}, \beta^{2}\right) \quad v_{2}\left(\beta^{1}, \beta^{2}\right) \quad v_{3}\left(\beta^{1}, \beta^{2}\right)\right]^{T}
$$

and the same NURBS basis functions used for the physical geometry discretization can be utilized to express the displacement field as

$$
\mathbf{v} \equiv \mathbf{v}\left(\beta^{1}, \beta^{2}\right)=\sum_{i} R_{i}(\xi, \eta) \mathbf{d}^{i} \equiv \sum_{i} R_{i} \mathbf{d}^{i}
$$

where $\mathbf{d}^{i}=\left[\begin{array}{lll}d_{1}^{i} & d_{2}^{i} & d_{3}^{i}\end{array}\right]^{T}$ is the translational DOFs of each control point that are used to define the local basis functions the iKLS element. According to the Kirchhoff-Love hypothesis as the director vector $\mathbf{F}$ remains normal to the mid-surface, deformation of any arbitrary point in the shell body can be defined from a linearized rotation $\boldsymbol{\theta}$ of the director vector $\mathbf{F}$ as [13]

$$
\mathbf{u} \equiv \mathbf{u}\left(\beta^{1}, \beta^{2}, \beta^{3}\right)=\mathbf{v}+\beta^{3}(\boldsymbol{\theta} \times \mathbf{F})
$$

where the rotation vector $\boldsymbol{\theta}$ represents an element of tangent space spanned by in-plane base vectors $\mathbf{a}_{\alpha}$ and can be defined as

$\boldsymbol{\theta} \equiv \boldsymbol{\theta}\left(\beta^{1}, \beta^{2}\right)=\phi_{1} \varphi_{1}+\phi_{2} \varphi_{2} \equiv\left(\mathbf{v}_{, 2} \cdot \mathbf{F}\right) \varphi_{1}-\left(\mathbf{v}_{, 1} \cdot \mathbf{F}\right) \varphi_{2}$

$\boldsymbol{\varphi}_{\alpha}=\frac{\mathbf{a}_{\alpha}}{\left\|\mathbf{a}_{1} \times \mathbf{a}_{2}\right\|}$

where $\boldsymbol{\varphi}_{\alpha}$ is the director of rotation angles $\phi_{\alpha}$. It is important to note that, the difference vector $\boldsymbol{\theta} \times \mathbf{F}$ represents the difference between the director of the reference and current configuration of the shell body. For small deformations, the linearized three-dimensional Green-Lagrange strain in curvilinear coordinates can be defined as

$\boldsymbol{\varepsilon}=\varepsilon_{i j} \mathbf{G}^{i} \otimes \mathbf{G}^{j}$

with

$$
\varepsilon_{i j}=\frac{1}{2}\left(\mathbf{u}_{, i} \cdot \mathbf{G}_{j}+\mathbf{u}_{, j} \cdot \mathbf{G}_{i}\right)
$$

where $\mathbf{G}^{i}$ is the contravariant base vectors of the shell and $\varepsilon_{i j}$ is the Green-Lagrange strain tensor coefficients. The individual components of Green-Lagrange strain tensor coefficients $\varepsilon_{i j}$ can be computed as

American Institute of Aeronautics and Astronautics 


$$
\begin{aligned}
& \varepsilon_{11}=\mathbf{v}_{, 1} \cdot \mathbf{a}_{1}+\beta^{3}\left(\mathbf{v}_{, 1} \cdot \mathbf{F}_{, 1}+\left(\mathbf{F} \times \mathbf{a}_{1}\right) \cdot \boldsymbol{\theta}_{, 1}\right) \\
& \varepsilon_{22}=\mathbf{v}_{, 2} \cdot \mathbf{a}_{2}+\beta^{3}\left(\mathbf{v}_{, 2} \cdot \mathbf{F}_{, 2}+\left(\mathbf{F} \times \mathbf{a}_{2}\right) \cdot \boldsymbol{\theta}_{, 2}\right) \\
& 2 \varepsilon_{12}=\gamma_{12}=\mathbf{v}_{, 1} \cdot \mathbf{a}_{2}+\mathbf{v}_{, 2} \cdot \mathbf{a}_{1}+\beta^{3}\left(\mathbf{v}_{, 1} \cdot \mathbf{F}_{, 2}+\mathbf{v}_{, 2} \cdot \mathbf{F}_{, 1}+\left(\mathbf{F} \times \mathbf{a}_{2}\right) \cdot \boldsymbol{\theta}_{, 1}+\left(\mathbf{F} \times \mathbf{a}_{1}\right) \cdot \boldsymbol{\theta}_{, 2}\right) \\
& \varepsilon_{i 3}=0
\end{aligned}
$$

For the sake of brevity, the non-zero Green-Lagrange strain tensor coefficients of iKLS element can be written in a compact vector form as

$$
\begin{aligned}
& \left\{\begin{array}{l}
\varepsilon_{11} \\
\varepsilon_{22} \\
\gamma_{12}
\end{array}\right\} \equiv \mathbf{e}\left(\mathbf{d}^{e}\right)+\beta^{3} \mathbf{k}\left(\mathbf{d}^{e}\right) \equiv \mathbf{B}^{m} \mathbf{d}^{e}+\zeta \mathbf{B}^{b} \mathbf{d}^{e} \\
& \mathbf{d}^{e}=\left[\begin{array}{lll}
\mathbf{d}^{1} & \mathbf{d}^{2} \quad \mathbf{d}^{N_{c p}}
\end{array}\right]^{T}
\end{aligned}
$$

where $\mathbf{e}\left(\mathbf{d}^{e}\right)$ is the membrane strains associated with stretching of the mid-surface, $\mathbf{k}\left(\mathbf{d}^{e}\right)$ is the bending curvatures, $\mathbf{d}^{e}$ is translational DOF of all the control points, and the superscript $N_{c p}$ is total number of control points. The matrices $\mathbf{B}^{m}$ and $\mathbf{B}^{b}$, given in Eq. (9a), contain the derivatives of the NURBS shape functions corresponding to membrane and bending actions of the iKLS element, respectively.

Discrete in-situ strain measurements obtained from on-board sensors are the fundamental input of the iFEM formulation. To calculate membrane and bending section strains experimentally, the in-situ strain rosettes should be located on top and bottom surfaces of the iKLS element, as shown in Figure 2.

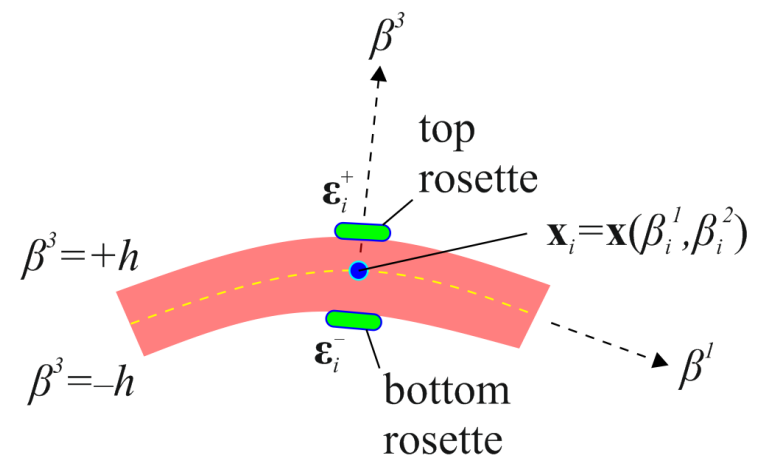

Figure 2. Discrete surface strains measured by strain rosettes within iKLS element at $\mathbf{X}_{i} \equiv \mathbf{X}\left(\beta_{i}^{1}, \beta_{i}^{2}, \pm h\right)$ locations.

The experimentally measured (in-situ) membrane section strains $\mathbf{e}_{i}^{\varepsilon}$ and curvatures $\mathbf{k}_{i}^{\varepsilon}$ that correspond to their analytic counterparts, $\mathbf{e}\left(\mathbf{d}^{e}\right)$ and $\mathbf{k}\left(\mathbf{d}^{e}\right)$ given by Eq. (9a), can be determined from the measured surface strains at $N_{s}$ discrete locations $\mathbf{X}_{i} \equiv \mathbf{X}\left(\beta_{i}^{1}, \beta_{i}^{2}, \pm h\right) \quad\left(i=1, \ldots, N_{s}\right)$ located within the element. These in-situ section strains are computed as follows $[1,2]$

$\mathbf{e}_{i}^{\varepsilon}=\frac{1}{2}\left(\boldsymbol{\varepsilon}_{i}^{+}+\boldsymbol{\varepsilon}_{i}^{-}\right)$

and

$\mathbf{k}_{i}^{\varepsilon}=\frac{1}{2 h}\left(\boldsymbol{\varepsilon}_{i}^{+}-\boldsymbol{\varepsilon}_{i}^{-}\right)$

where

American Institute of Aeronautics and Astronautics 
$\boldsymbol{\varepsilon}_{i}^{+}=\left[\begin{array}{lll}\varepsilon_{11}^{+} & \varepsilon_{22}^{+} & \gamma_{12}^{+}\end{array}\right]_{i}^{T}$

and

$\boldsymbol{\varepsilon}_{i}^{-}=\left[\begin{array}{lll}\varepsilon_{11}^{-} & \varepsilon_{22}^{-} & \gamma_{12}^{-}\end{array}\right]_{i}^{T}$

are the measured surface strains, with the superscripts ' + ' and '-' denoting the quantities that correspond to the top and bottom surface locations, respectively.

The iFEM formulation reconstructs the deformed shape of a discretized structure by minimizing a least-squares functional with respect to the nodal DOF of the entire discretization. For an individual iKLS element, this functional, $\Phi\left(\mathbf{d}^{e}\right)$, accounts for the membrane and bending deformations and can be expressed as

$\Phi\left(\mathbf{d}^{e}\right)=\left\|\mathbf{e}\left(\mathbf{d}^{e}\right)-\mathbf{e}^{\varepsilon}\right\|^{2}+\left\|\mathbf{k}\left(\mathbf{d}^{e}\right)-\mathbf{k}^{\varepsilon}\right\|^{2}$

The squared norms expressed in Eq. (11a) can be written in the form of the normalized Euclidean norms as

$\left\|\mathbf{e}\left(\mathbf{d}^{e}\right)-\mathbf{e}^{\varepsilon}\right\|^{2} \equiv \frac{1}{N_{s}} \int_{A}^{N_{s}} \sum_{i=1}\left[\mathbf{e}\left(\mathbf{d}^{e}\right)_{i}-\mathbf{e}_{i}^{\varepsilon}\right]^{2} d A$

and

$\left\|\mathbf{k}\left(\mathbf{d}^{e}\right)-\mathbf{k}^{\varepsilon}\right\|^{2} \equiv \frac{(2 h)^{2}}{N_{s}} \int_{A}^{N_{s}} \sum_{i=1}\left[\mathbf{k}\left(\mathbf{d}^{e}\right)_{i}-\mathbf{k}_{i}^{\varepsilon}\right]^{2} d A$

where $A$ represents the mid-plane area of the element. Minimizing the iFEM least-squares functional, given by Eq. (11a), with respect to the nodal displacement DOF gives rise to

$\delta \Phi\left(\mathbf{d}^{e}\right) \equiv \frac{\partial \Phi\left(\mathbf{d}^{e}\right)}{\partial \mathbf{d}^{e}}=\Gamma^{e} \mathbf{d}^{e}-\mathbf{E}^{e}=0$

or simply

$\Gamma^{e} \mathbf{d}^{e}=\mathbf{E}^{e}$

where $\Gamma^{e}$ is the local left-hand-side matrix, $\mathbf{E}^{e}$ is the local right-hand-side vector that is a function of the measured strain values, and $\mathbf{d}^{e}$ is translational DOF of all the control points that are the unknowns in the analysis. The element $\boldsymbol{\Gamma}^{e}$ matrix can be explicitly written in terms of the matrices $\mathbf{B}^{m}$ and $\mathbf{B}^{b}$ as

$\boldsymbol{\Gamma}^{e}=\int_{A}\left[\left(\mathbf{B}^{m}\right)^{T} \mathbf{B}^{m}+(2 h)^{2}\left(\mathbf{B}^{b}\right)^{T} \mathbf{B}^{b}\right] d A$

The $\mathbf{E}^{e}$ vector is a function of the number of strain sensors within the element as well as the measured section-strain values, and is given as

$\mathbf{E}^{e}=\frac{1}{N_{s}} \int_{A} \sum_{i=1}^{N_{s}}\left[\left(\mathbf{B}^{m}\right)^{T} \mathbf{e}_{i}^{\varepsilon}+(2 h)^{2}\left(\mathbf{B}^{b}\right)^{T} \mathbf{k}_{i}^{\varepsilon}\right] d A$

Once the element matrix equations are established, the element contributions to the global linear equation system of the discretized structure can be evaluated. After that, the resulting system of equations can be reduced to wellconditioned system of equations by prescribing problem-specific displacement boundary conditions. The solution of this system of equations provides the deformed structural shape at any real-time.

\section{Numerical results}

\section{The pinched hemisphere}

The pinched hemisphere problem proposed and studied by many researchers $[14,15]$ is solved here as a benchmark problem. The hemispheric shell is subjected to the four different concentrated loads as shown in Figure 3.

American Institute of Aeronautics and Astronautics 
The prescribed boundary conditions are the minimum required to prevent rigid body motions. In other words, the apex of the hemisphere along $Z$-direction needs to be fixed in order to eliminate the rigid body motion. Linear, elastic and isotropic material properties $E$ and $v$, magnitude of the concentrated force $F$, radius of the hemisphere $R$, and thickness of the hemisphere $2 h$ are given in Figure 3. This problem is challenging in terms of demonstrating bending capability of the iKLS element because it exhibits almost none of membrane strains. Moreover, doubly curved geometry and point loading make this problem highly sensitive to locking phenomena. By utilizing the advantage of symmetry, only one quarter of the structure is modelled and symmetry constraint and loading boundary conditions are applied. The quantity of interest is $U_{A}$, i.e., the displacement along the direction of the loading $F$ at point $A$ (refer to Figure 3 ). The associated reference solution is given as $U_{A}=0.0924 \mathrm{~m}$ in $[14,15]$.

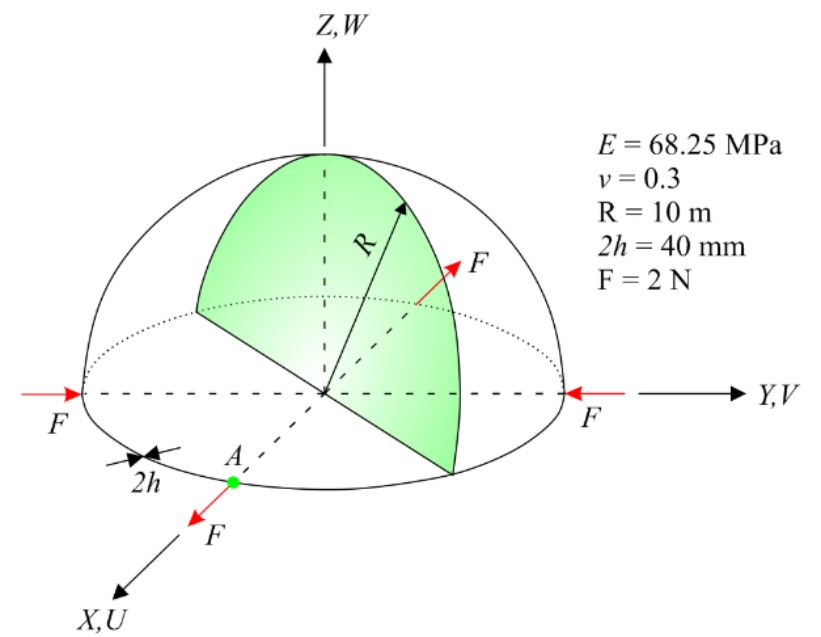

Figure 3. Pinched hemisphere.

An accurate reference solution is established by performing a convergence study using direct FEM analysis. The most refined mesh consisted of 7500 uniformly distributed rectangular elements, possessing $45906 \mathrm{DOF}$. The value of the displacement at point $A$ is found as $U_{A}=0.0921 \mathrm{~m}$ from the high-fidelity FEM analysis, which is in a fairly well agreement with the reference solution. Therefore, the FEM deflections and rotations are used to calculate the simulated strain-sensor strains. In the current iFEM analysis, five different iKLS discretization are generated by uniformly dividing edges of one quarter of the hemisphere into 2, 4, 6, 8, and 10 segments, respectively. In each iKLS mesh, two strain rosettes are placed at the centroid of each iKLS element, one on the top and the other on the bottom surface. According to this arrangement of strain sensors, all of the iKLS elements are installed with strain sensors for each iFEM analysis. An example of strain rosette configuration for 4 segments per edge is illustrated for the iKLS discretization in Figure 4.

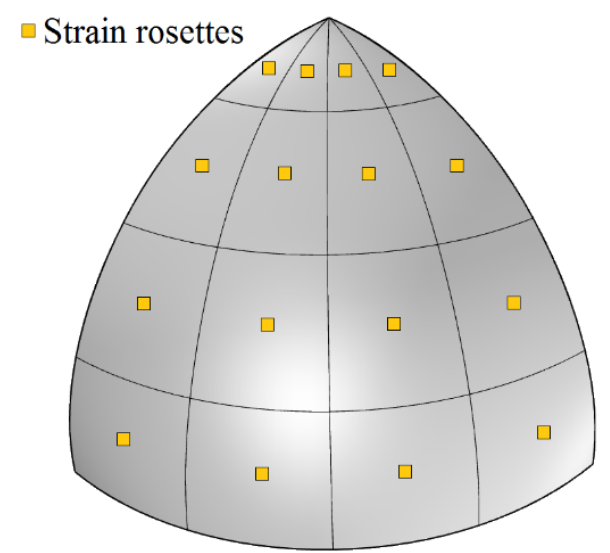

Figure 4. Discretization (4 segments per edge) of one-fourth of the hemisphere using iKLS elements with topand bottom-surface strain rosettes per each element. 
Once the iFEM analyses are performed using iKLS model, the same shape sensing analyses are also conducted using the iQS4 element [4] in order to make a clear comparison between iKLS and QQS4 elements. The $U_{A}$ displacement results are plotted with respect to the number of sensors on the each edge, as depicted in Figure 5. This result clearly demonstrates the greater shape sensing accuracy of the iKLS element in comparison to iQS4 element. In fact, using iKLS element allows us to improve the accuracy of shape sensing analysis even if a very coarse mesh (with a low number of strain-sensors) is used for the analysis. This is due to the fact that the polynomial degree of the NURBS can be elevated without changing the location of knots, hence the number of elements will remain unchanged. This feature of the NURBS basis function can be exploited for obtaining more accurate results.

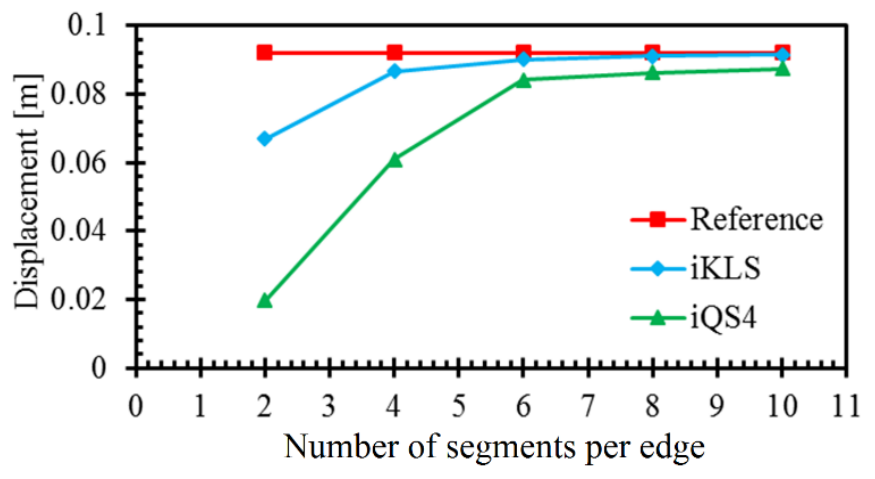

Figure 5. $U_{A}$ displacement result comparison between iKLS and iQS4 elements.

The contour plots of the $U$ displacement are depicted in Figure 6 where contour plots for iFEM analysis are graphically almost identical to those of FEM analysis. Moreover, the percent difference between iFEM and FEM predictions for maximum value of the $U$ displacement is approximately $5.8 \%$ (refer to Figure 6 ). These observations confirm the superior bending capability of the iKLS element, even if a low-fidelity discretization (4 segments per edge) with few number of sensors (i.e., $16 \times 2=32$ strain rosettes in total) is used in the shape sensing analysis. Remarkably, these predictions demonstrate the high quality precision of isogeometric iFEM solutions for shape sensing analysis of a complex and doubly curved geometry.
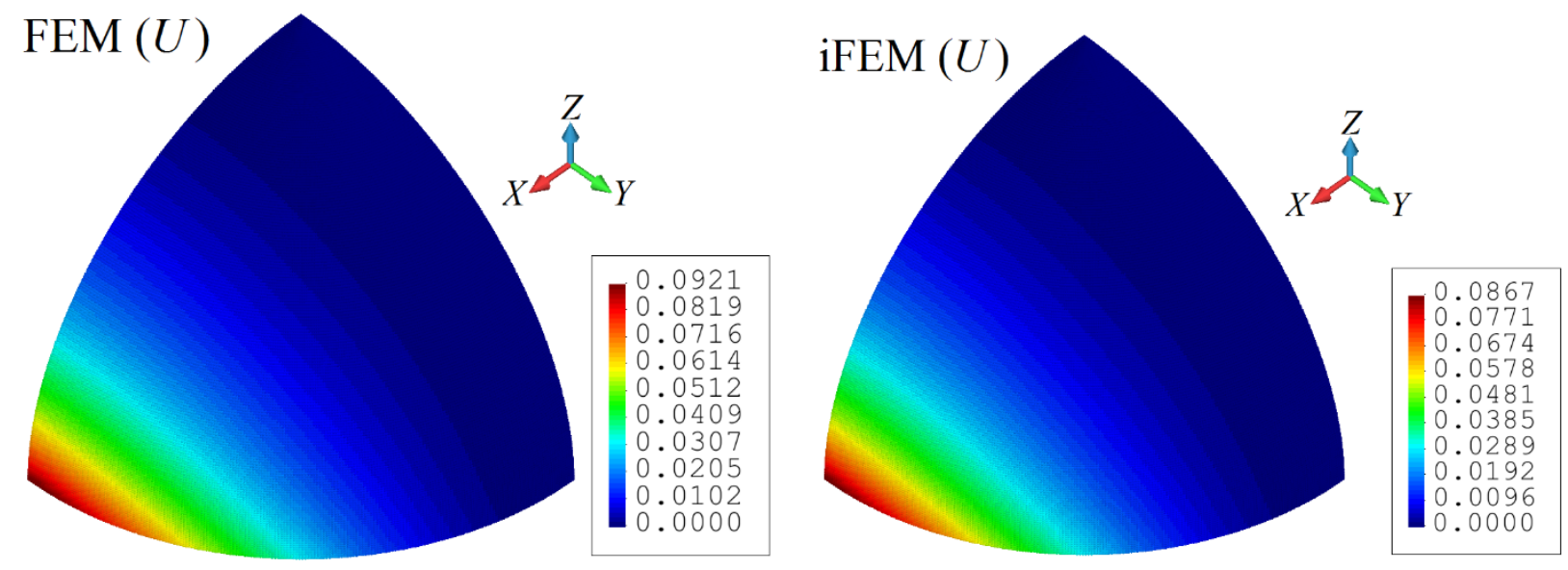

Figure 6. Contour plots of $U$ displacement for iKLS model depicted in Figure 4; comparison between highfidelity FEM and iFEM analyses.

\section{A partly clamped hyperbolic paraboloid}

According to Bathe and co-workers [16], partly clamped hyperbolic paraboloid problem is an excellent test for locking in bending-dominated situations. Therefore, shape sensing of the hyperbolic paraboloid is performed using 
the iKLS element in order to better assess the capability of the iKLS element against the locking phenomenon. The mid-surface of the hyperbolic paraboloid is defined as

$$
Z=X^{2}-Y^{2},(X, Y) \in[-L / 2 ; L / 2]
$$

It is very straightforward to construct this surface using second order B-splines. As depicted in Figure 7, the shell is clamped from the edge located at $X=-L / 2$ and a gravitational load of $g$ is applied. Linear, elastic and isotropic material properties and exact geometry of the shell is also given in Figure 7. Utilizing the symmetry of the problem, only one half of the shell is modelled for the computation and relevant symmetry constraint boundary conditions are applied. The quantity of interest is $W_{A}$, i.e., the vertical displacement along $Z$-direction at point $A$ which is the midpoint of the edge located at $X=+L / 2$, as shown in Figure 7. The associated reference solution is found as $W_{A}=-6.3941 \mathrm{~mm}$ in [16].

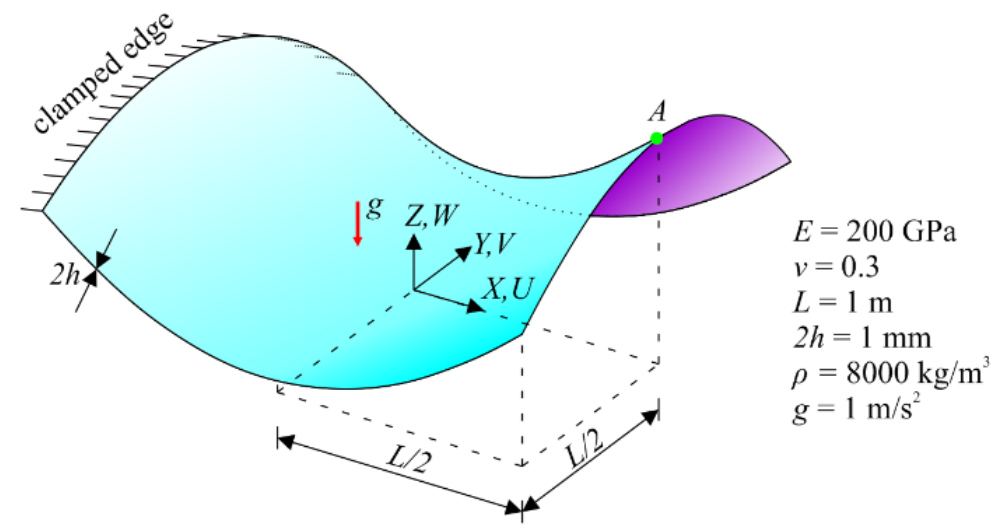

Figure 7. Hyperbolic paraboloid.

A convergence study was performed using direct FEM analysis and an accurate reference solution is established for simulating sensor strains. The highest fidelity mesh has 22500 uniformly distributed rectangular elements and 136806 DOF. The high-fidelity FEM analysis predicts this vertical displacement as $W_{A}=-6.4061 \mathrm{~mm}$ which agrees well with its associated reference solution [16]. In the following iFEM analysis, this problem is analyzed using five different iKLS discretization where edges of the geometry are uniformly divided into 2, 4, 6, 8, and 10 segments, respectively. Similar to the previous case study (hemisphere problem), each iKLS element has two strain rosettes in each iKLS model. Therefore, five different iFEM analysis of the paraboloid are performed considering all the iKLS elements installed with strain sensors. To give an example of strain rosette configurations, an iKLS model with 6 segments per edge is presented in Figure 8.

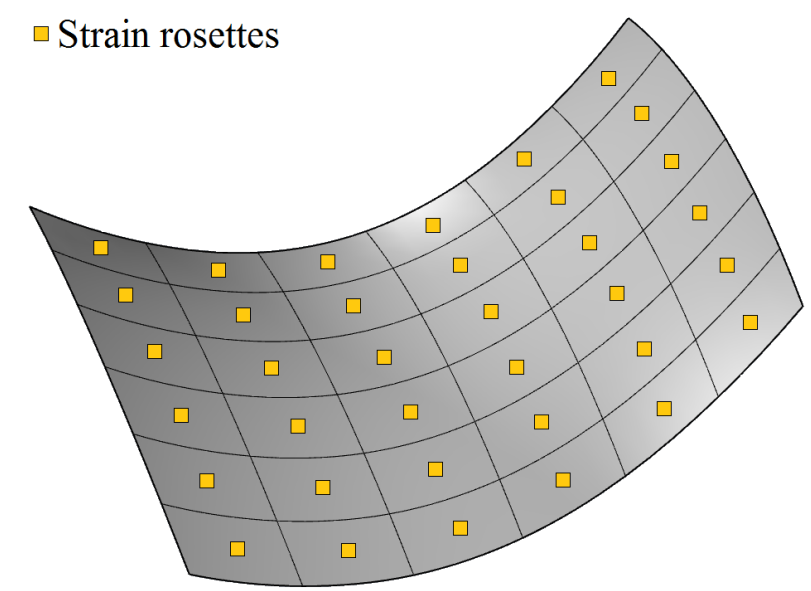

Figure 8. Discretization (6 segments per edge) of half of the hyperbolic paraboloid using iKLS elements with top- and bottom-surface strain rosettes per each element.

American Institute of Aeronautics and Astronautics 
Apart from the iKLS/iFEM analysis, the same shape sensing analyses of the hyperbolic paraboloid are also conducted using the iQS4 models with same number of strain sensors per edge. As depicted in Figure 9, the $\left|W_{A}\right|$ displacement results obtained using iKLS and iQS4 elements are compared for varying number of sensors. Remarkably, this result validates the superior shape sensing capability of the iKLS element.

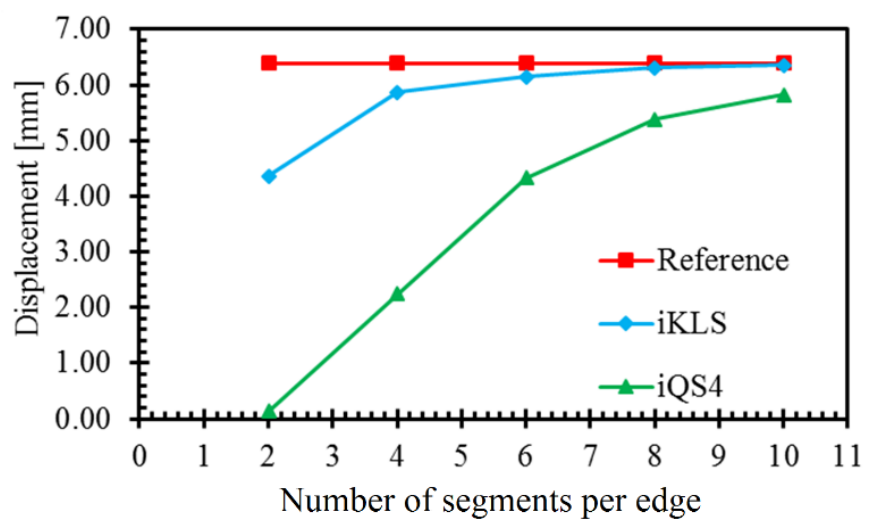

Figure 9. $\left|W_{A}\right|$ displacement result comparison between iKLS and iQS4 elements.

Furthermore, in Figures 10, contour plots of $W$ displacements are compared between the iFEM and high-fidelity FEM analyses. In these figures, the iFEM contours correspond to the iFEM analysis that uses the iKLS model (6 segments as presented in Figure 8) with $36 \times 2$ strain rosettes in total. The percent difference between the iFEM and FEM for the maximum values of the $W$ displacement is only $4.1 \%$ (refer to Figure 10). As can be seen from these plots, both the iFEM and direct FEM contours are graphically indistinguishable. These results demonstrate the superior bending predictions of iKLS element, especially considering the low-fidelity mesh used in the iFEM analysis.
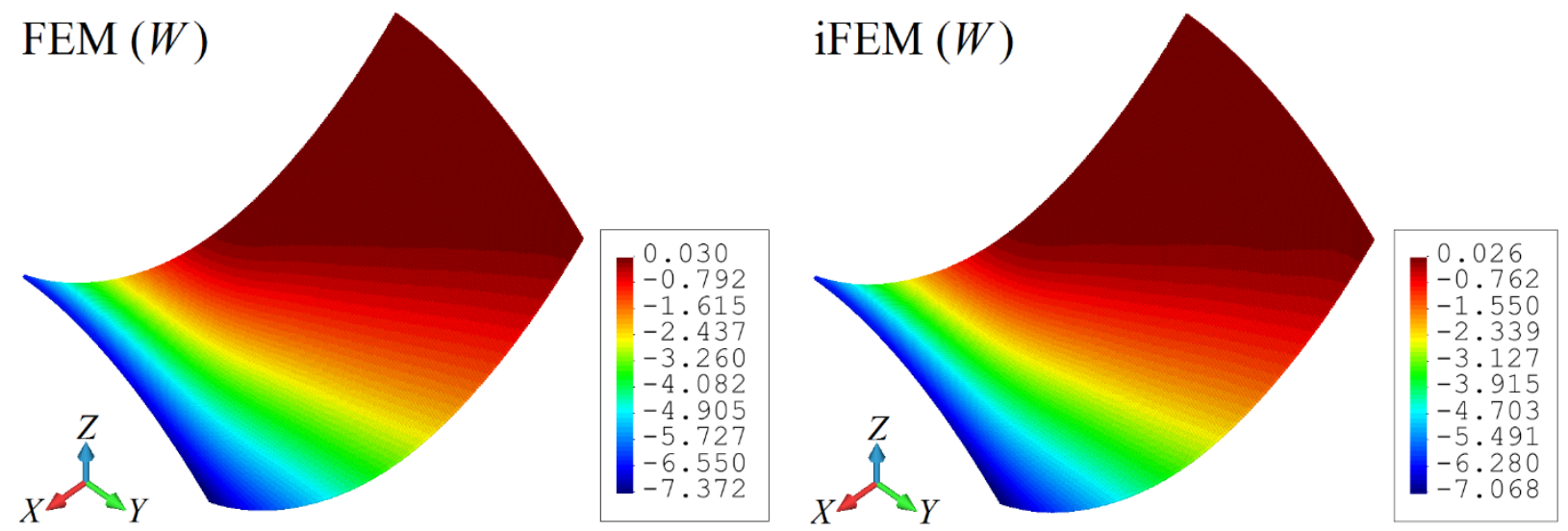

Figure 10. Contour plots of $\bar{W}$ displacement for iKLS model depicted in Figure 8; comparison between highfidelity FEM and iFEM analyses.

\section{Conclusions}

This paper focuses on the shape sensing of complex shell structures by coupling the NURBS-based IGA together with the iFEM methodology. An isogeometric Kirchhoff-Love inverse-shell element (iKLS) is developed for the numerical implementation of the proposed coupling between IGA and iFEM (isogeometric iFEM methodology). The presented iKLS formulation is applicable to perform shape-sensing analyses of complex thin plate and shell structures by using the strain data obtained from randomly distributed sensors on the structure. The bending capability of the iKLS element is examined by carrying out two numerical simulations including a pinched hemisphere and a partly clamped hyperbolic paraboloid. As a result, the bending efficiency of iKLS element has been justified even using the low-fidelity iKLS discretization. It has been demonstrated that the iKLS element has the advantage for shape sensing 
of complex shell structures in comparison to iQS4 element. Moreover, it has been confirmed that even with a very coarse mesh (with a low number of strain-sensors), the iKLS element provides improved results by increasing the polynomial degree of the NURBS shape function without changing the location of knots. This feature can be exploited in the future to obtain more accurate shape sensing results for different type of elements that will be developed based on the proposed coupling.

\section{References}

[1] Tessler, A., and Spangler, J. L., "A variational principal for reconstruction of elastic deformation of shear deformable plates and shells," NASA TM-212445, 2003.

[2] Tessler, A., and Spangler, J. L., "A least-squares variational method for full-field reconstruction of elastic deformations in shear-deformable plates and shells," Computer Methods in Applied Mechanics and Engineering, Vol. 194, No. 2, 2005, pp. $327,339$.

[3] Tessler, A., and Spangler, J. L., "Inverse FEM for full-field reconstruction of elastic deformations in shear deformable plates and shells," Proceedings of 2nd European Workshop on Structural Health Monitoring, Munich, Germany, 2004.

[4] Kefal, A., Oterkus, E., Tessler, A., and Spangler, J. L., "A quadrilateral inverse-shell element with drilling degrees of freedom for shape sensing and structural health monitoring," Engineering Science and Technology, an International Journal, Vol. 19, 2016, pp. 1299, 1313.

[5] Gherlone, M., Cerracchio, P., Mattone, M., Di Sciuva, M., and Tessler, A., "Shape sensing of 3D frame structures using an inverse Finite Element Method," International Journal of Solids and Structures, Vol. 49, No. 22, 2012, pp. $3100,3112$.

[6] Cerracchio, P., Gherlone, M., Di Sciuva, M., and Tessler, A., "A novel approach for displacement and stress monitoring of sandwich structures based on the inverse Finite Element Method," Composite Structures, Vol. 127, 2015, pp. 69, 76.

[7] Tessler, A., Di Sciuva, M., and Gherlone, M., "A consistent refinement of first-order shear deformation theory for laminated composite and sandwich plates using improved zigzag kinematics," Journal of Mechanics of Materials and Structures, Vol. 5, No. 2, 2010, pp. 341, 367.

[8] Kefal, A., and Oterkus, E., "Structural Health Monitoring of marine structures by using inverse Finite Element Method," Analysis and Design of Marine Structures V, 2015, pp. 341-349.

[9] Kefal, A., and Oterkus, E., "Displacement and stress monitoring of a chemical tanker based on inverse finite element method," Ocean Engineering, Vol. 112, 2016, pp. 33, 46.

[10] Kefal, A., and Oterkus, E., "Displacement and stress monitoring of a Panamax containership using inverse finite element method," Ocean Engineering, Vol. 119, 2016, pp. 16, 29.

[11] Hughes, T. J., Cottrell, J. A., and Bazilevs, Y., "Isogeometric analysis: CAD, finite elements, NURBS, exact geometry and mesh refinement," Computer Methods in Applied Mechanics and Engineering, Vol. 194, No. 39, 2005, pp. 4135, 4195.

[12] Cottrell, J. A., Hughes, T. J., and Bazilevs, Y., Isogeometric Analysis: Toward Integration of CAD and FEA, 2009, John Wiley and Sons.

[13] Echter, R., Oesterle, B., and Bischoff, M, "A hierarchic family of isogeometric shell finite elements," Computer Methods in Applied Mechanics and Engineering, Vol. 254, 2013, pp. 170, 180.

[14] MacNeal, R. and Harder, R., "A proposed standard set of problems to test finite element accuracy," Finite Elements in Analysis and Design, Vol. 1, 1985, pp. 3, 20.

[15] Belytschko, T., Stolarski, H., Liu, W., Carpenter, N., and Ong, J. S., "Stress projection for membrane and shear locking in shell finite elements," Computer Methods in Applied Mechanics and Engineering, Vol. 51, No. 1, 1985, pp. $221,258$.

[16] Bathe, K. J., Iosilevich, A., and Chapelle, D., "An evaluation of the MITC shell elements," Computers and Structures, Vol. 75 , No. 1, 2000, pp. 1, 30 .

American Institute of Aeronautics and Astronautics 